\title{
Neoplasia intraepitelial prostática aislada y positividad para adenocarcinoma en la rebiopsia. Revisión de nuestra serie
}

\author{
M. Arzoz Fábregas, J. Areal Calama, L. Ibarz Servio, J.L. Gago Ramos, R. Boix Orri, \\ J.Mํㅗㄴ Saladié Roig
}

Servicio de Urología. Hospital Germans Trias i Pujol. Badalona. Barcelona.

Actas Urol Esp 2005; 29 (8): 735-738

\section{RESUMEN}

\section{NEOPLASIA INTRAEPITELIAL PROSTÁTICA AISLADA Y POSITIVIDAD PARA ADENOCARCINOMA EN LA REBIOPSIA.} REVISIÓN DE NUESTRA SERIE

Objetivo: Revisar la incidencia de neoplasia intraepitelial prostática ( PIN ) aislada, así como la positividad para adenocarcinoma prostático de estas lesiones en la rebiopsia en nuestra serie de biopsias transrectales, comparando los resultados con la bibliografía actual.

Material y Método : Hemos analizado las 2.475 biopsias prostáticas transrectales ecodirigidas realizadas en nuestro Servicio desde enero de 1992 hasta junio del 2004 en búsqueda de neoplasia intraepitelial prostática y en concreto de PIN de alto grado (PINAG) aislado, revisando las rebiopsias realizadas a estos pacientes y la probabilidad de detectar en ellas adenocarcinoma.

Resultados: De un total de 31 biopsias en las que se halló el diagnóstico de PIN, 13 de ellas corresponde a PIN de alto grado aislado, lo que supone una incidencia de $0,52 \%$ del total de las biopsias realizadas en el Servicio. Se han rebiopsiado 7 de estos 13 casos en un período de 3 meses a 2 años tras la primera biopsia, hallando en 3 adenocarcinoma; lo que supone que el $43 \%$ de los PINAG aislados rebiopsiados en la serie mostraron adenocarcinoma. La edad media de los pacientes estudiados fue de 67 años (rango 53-88). El PSA medio de los pacientes estudiados fue de 9,8 ng/ml. El Gleason de las biopsias con carcinoma estuvo siempre entre 3 y 7 . En nuestra muestra, la edad y los niveles de PSA no guardaban relación con la positividad a adenocarcinoma en las rebiopsias.

Conclusiones: A pesar de una cifra inferior de PIN de alto grado en nuestra serie, la positividad para carcinoma en la rebiopsia (43\%) es semejante a la descrita en la literatura, por lo que son aconsejables los controles rigurosos y las rebiopsias a estos pacientes.

Palabras clave: Neoplasia intraepitelial prostática. Biopsia prostática. Cáncer de próstata.

\section{ABSTRACT}

ISOLATED PROSTATIC INTRAEPITHELIAL NEOPLASIA AND POSITIVE PROSTATE CANCER RESULTS AT REPEAT BIOPSY. OUR SERIES REVIEW

Objetive: To review the incidence of isolated prostatic intraepithelial neoplasia (PIN) as well as the positive prostate cancer results in repeat biopsy in our series of transrectal biopsy of the prostate. We compare these results with the actual literature.

Materials and Methods: We review the 2.475 transrectal ultrasound guided biopsies of the prostate made in our department from January 1992 to June 2004 looking for intraepithelial neoplasia and looking in particular for isolated High-grade PIN ( HGPIN). We review repeat biopsies made at this patients and the likelihood of detecting prostate cancer in them.

Results: The diagnosis of PIN was found in 31 biopsies, 13 of them were isolated HGPIN what means a $0,52 \%$ incidence of the total biopsies performed in our department. We performed 7 repeat biopsies of the 13 cases in a period from 3 months to 2 years after the first biopsy, and we found 3 cases of prostate cancer what means that $43 \%$ of isolated HGPIN with repeated biopsy showed prostate cancer. The mean age of the patients studied was 67 years (range 53-88). The median PSA value of the studied cases was $9,8 \mathrm{ng} / \mathrm{ml}$. The Gleason score of the positive biopsies was always between 3 and 7 . In our series, age and PSA levels did not have correlation with the positive results for prostate cancer in repeated biopsies.

Conclusions: In spite of a lower number of HGPIN cases in our series, the positive for prostate cancer in repeated biopsy ( $43 \%$ ) is similar to the incidence reported in literature so it is advisable rigorous controls and repeated biopsies for these patients.

Keywords: Prostatic Intraepithelial Neoplasia. Prostate biopsy. Prostate cancer. 
$\mathrm{E}$ 1 término de Neoplasia intraepitalial prostática (PIN) es introducido por Bostwick y Brawer a finales de los ochenta para describir un conjunto de alteraciones arquitecturales y citológicas del epitelio de los ductos prostáticos ${ }^{1,2}$. Inicialmente, Mc Neal y Bostwick describieron tres grados de PIN 1, 2 y 3, según la gravedad de las alteraciones del epitelio ductal ${ }^{3}$. En la actualidad, hablamos de PIN de bajo grado (PIN1) o bien PIN de alto grado (PIN 2 y 3), siendo ésta clasificación de gran relevancia ya que el hallazgo de un PIN de alto grado aislado (PINAG) se considera el más importante precursor de adenocarcinoma prostático. Por contra, se considera que el PINBG tiene un bajo valor predictivo como precursor de adenocarcinoma y que, existe mucha variabilidad interobservador al informar este tipo de lesiones ${ }^{4,5}$.

Según la literatura, la frecuencia del PINAG en las biopsias prostáticas oscila desde 0,7-15\% dependiendo ésta de factores como la población estudiada y los criterios de indicación de biop$\mathrm{sia}^{6,7}$. Un $40 \%$ de las biopsias con PINAG coinciden con la presencia de adenocarcinoma de próstata en la misma biopsia, existiendo en las biopsias con PINAG aislado un riesgo de $30-100 \%$ (con una media del 50\%) de diagnosticar un carcinoma de próstata en la rebiopsia ${ }^{7}$.

\section{MATERIAL Y MÉTODOS}

Hemos analizado nuestra serie de biopsias prostáticas transrectales ecodirigidas, un total de 2.475 biopsias desde 1992 a junio del 2004, realizadas a petición de facultativos especialistas de nuestro centro (el $52 \%$ de las biopsias practicadas) y especialistas del área primaria (el $48 \%$ restante de biopsias) a pacientes con niveles de $\mathrm{PSA} \geq 4 \mathrm{ng} / \mathrm{ml}$ y/o tacto rectal sospechoso.

En ausencia de hallazgos ecográficos, la mayoría de biopsias se realizaron según la técnica de punción por sextantes. Los pacientes se biopsiaban en el área de consultas externas, de forma ambulatoria y bajo profilaxis con ciprofloxacino oral. Se utilizaba una pistola automática Biopty ${ }^{\circledR}$, con agujas de 18 g. La preparación incluía sistemáticamente un enema de limpieza de $250 \mathrm{ml}$, pero no anestésicos ni ansiolíticos.

Se revisó retrospectivamente la presencia del diagnóstico de PIN en la base de datos de biopsias.
Se seleccionó la historia de 13 pacientes por presentar PINAG aislado, analizando las rebiopsias realizadas a estos pacientes. También se estudió la posible influencia del nivel de PSA y la edad sobre la progresión a adenocarcinoma en estos pacientes mediante análisis univariante. Utilizamos el paquete informático SPSS 11.0 para Windows; en concreto la prueba U Mann Whithey para variables cuantitativas y el test de Fisher para las cualitativas.

\section{RESULTADOS}

De un total de 2.475 biopsias, hemos encontrado 31 biopsias $(1,25 \%)$ practicadas a 30 pacientes en las que aparece el término PIN $(1,2,3$, de bajo o alto grado). Detectamos 5 biopsias con PINBG aislado y otro PINBG con adenocarcinoma. En 12 de las biopsias con PIN, la presencia de PINAG coexistía con la de adenocarcinoma prostático en la misma biopsia $(38,7 \%)$ y en 13 de las biopsias (Fig. 1) se halló PINAG en uno o ambos lóbulos de forma aislada (42\%), lo que corresponde al $0,52 \%$ del total de las biopsias realizadas. Se han rebiopsiado 7 de estos 13 casos de PINAG aislado en un periodo de 3 meses a 2 años, hallando 3 pacientes con rebiopsias positivas para adenocarcinoma $(42,8 \%)$, las cuatro rebiopsias restantes se informaron como benignas, sin repetirse de nuevo el diagnóstico de PINAG aislado. Tres pacientes se sometieron a una segunda rebiopsia con resultado negativo para adenocarcinoma. El tiempo medio de la rebiopsia fue de 12 meses con un mínimo de 3 meses y un máximo de 24 meses. Aunque nuestro criterio es el de la rebiopsia sistemática, es preciso mencionar que, ocho de estas trece biopsias con PINAG aislado, fueron solicitadas por urólogos del área primaria y las cinco restantes las indicaron facultativos de nuestro servicio. De las 7 rebiopsias, cinco corresponden a los cinco pacientes controlados en nuestro servicio y las dos restantes pertenecen al grupo de pacientes del área primaria. No disponemos de información de cómo fue el seguimiento de los seis pacientes restantes, que pertenecían al área.

La edad media de los 31 pacientes fue de 67 años (53-88 años). La edad media de los pacientes con PINAG aislado fue de 66 años, siendo la media de edad de las rebiopsias con positividad a 


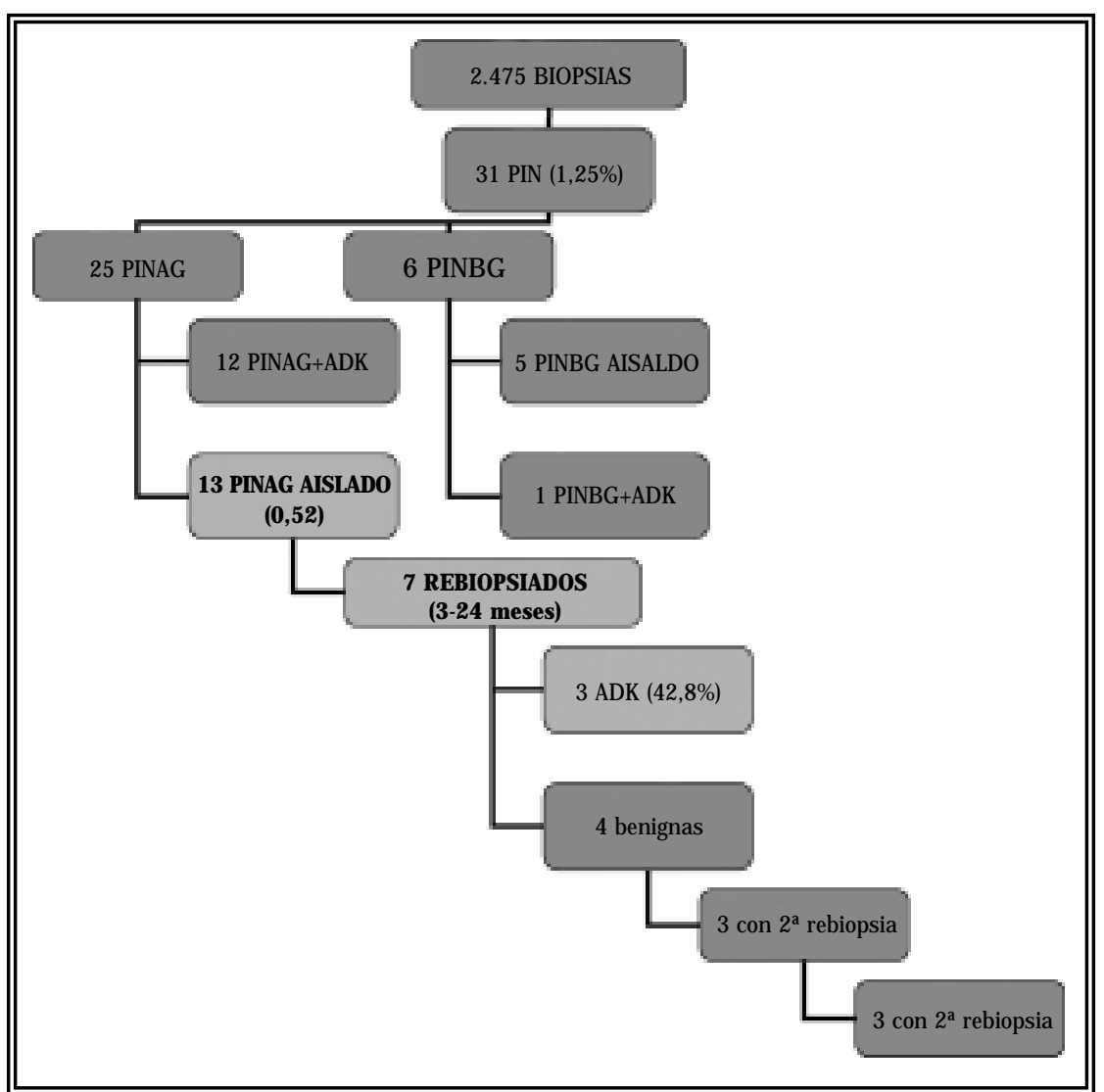

FIGURA 1. Neoplasia intraepitelial hallada en nuestra serie.

ADK de 70 años y de 61 años para las rebiopsias sin evidencia de neoplasia (Tabla 1). No encontramos relación estadísticamente significativa entre la edad y la positividad para adenocarcinoma en las rebiopsias de PINAG aislado $(p=0,62)$.

El PSA medio de los 31 pacientes estudiados fue de $10,2 \mathrm{ng} / \mathrm{ml}$. El PSA medio de los pacientes con PINAG aislado fue de 9`8, con una media de
PSA de 16 para las rebiopsias positivas para ADK y una media de PSA de 9,8 para las rebiopsias sin evidencia de neoplasia (Tabla 1). Al igual que con la edad, no encontramos relación estadísticamente significativa entre los niveles de PSA y la positividad para ADK en las rebiopsias realizadas $(p=0,31)$. Al estratificar los niveles de PSA por grupos según el percentil 75 $(\mathrm{PSA} \leq 9,5 \mathrm{ng} / \mathrm{ml}, \mathrm{PSA}>9,5 \mathrm{ng} / \mathrm{ml}$ ), tampoco encontramos relación estadísticamente significativa entre estos dos grupos y la positividad a adenocarcinoma en la rebiopsia $(p=0,14)$.

El Gleason de los quince casos, de los 31 estudiados, en los que aparece adenocarcinoma, ya sea concomitante con PINAG o bien en las rebiopsias positivas, estuvo siempre entre 3 y 7 (Tabla 1).

\section{DISCUSIÓN}

La cifra de PINAG aislado en nuestra serie es más baja que la hallada en la mayoría de la literatura (0,7-15\%), aunque se acerca a la detectada en revisones similares ${ }^{6,8}$. Por otro lado, el número de casos de PINAG que coexisten con cáncer en la biopsia o el número de casos rebiopsiados por PINAG aislado en los que hallamos carcinoma se asemeja más a los valores encontrados en otras series ${ }^{6,7,9}$. En nuestro cen-

Tabla 1

Medias de Edad, PSA y Gleason de los casos revisados

\begin{tabular}{|c|c|c|c|c|c|}
\hline & $\begin{array}{c}\text { Total } \\
\text { PIN } \\
\text { (31 casos) }\end{array}$ & $\begin{array}{c}\text { PINAG } \\
\text { aislado } \\
\text { (13 pacientes) }\end{array}$ & $\begin{array}{l}\text { PINAG más } \\
\text { ADK } \\
\text { (12 pacientes) }\end{array}$ & $\begin{array}{c}\text { Rebiopsia } \\
\text { positiva } \\
\text { (3 pacientes) }\end{array}$ & $\begin{array}{c}\text { Rebiopsia } \\
\text { negativa } \\
\text { (4 pacientes) }\end{array}$ \\
\hline $\begin{array}{l}\text { Edad } \\
\text { (años) }\end{array}$ & 67 & 66 & 69 & 70 & 61 \\
\hline $\begin{array}{l}\text { PSA } \\
\text { (ng/ml) }\end{array}$ & 10 & 9,8 & 10 & 16 & 9,8 \\
\hline \multirow[t]{2}{*}{$\begin{array}{l}\text { Gleason } \\
\text { score }\end{array}$} & & & $3-7$ & $4-7$ & \\
\hline & & & $\begin{array}{l}34 \% \text { Gleason } 3-4 \\
66 \% \text { Gleason } 5-7\end{array}$ & $\begin{array}{l}2 \text { casos Gleason } 4 \\
1 \text { caso Gleason } 7\end{array}$ & \\
\hline
\end{tabular}


tro, se informa cada vez menos de la presencia de PIN de bajo grado por su escasa significación clínica. Hemos de tener en cuenta también que se asocia a lesiones benignas y que su identificación es muy subjetiva ${ }^{2,10}$.

La edad media de los pacientes estudiados es parecida a la media de edad descrita en otras series $^{9,11,12}$ y al igual que la mayoría de los estudios no encontramos relación entre la edad y la progresión a adenocarcinoma en los pacientes con PINAG aislado.

Los niveles de PSA medio encontrados en las biopsias con PINAG aislado se asemejan al de otros estudios ${ }^{10}$. Aunque en el análisis descriptivo observamos una tendencia al alza del PSA medio en las rebiopsias positivas para adenocarcinoma, no evidenciamos relación estadísticamente significativa entre los niveles de PSA y la probabilidad de detectar cáncer en la rebiopsia. A pesar de que hay estudios en los que observan una relación significativa entre los niveles de PSA (y PSA libre) con el hallazgo de cáncer en la rebiopsia ${ }^{2,5}$, la mayoría de los estudios no han encontrado relación significativa ${ }^{7,9,10}$.

Actualmente se acepta el hecho de que el PINAG aislado es una lesión preneoplásica con un alto valor predictivo positivo como marcador de adenocarcinoma prostático. En la literatura se discute poco la necesidad de mantener una vigilancia e indicar rebiopsias a estos pacientes. A pesar de ello, no existe un acuerdo de cuál es el mejor manejo para estos casos y durante cuánto tiempo es necesario continuar con las rebiop$\operatorname{sias}^{7}$. En nuestro caso, el tiempo medio hasta la primera rebiopsia fue bastante más elevado que el de otras series estudiadas ${ }^{9}$. Existen estudios en los que no encuentran diferencias en la progresión a adenocarcinoma según se trate del resultado de la primera rebiopsia o de las subsiguientes biopsias. También hay autores que apuntan que si no se halla cáncer en las primeras dos rebiopsias es poco probable que se llegue a encontrarlo en posteriores exámenes ${ }^{9,12}$. Ninguna de nuestras segundas rebiopsias fueron positivas para adenocarcinoma.

\section{CONCLUSIONES}

A pesar de haber hallado una cifra inferior de PIN de alto grado en nuestra serie, a la hora de analizar su trascendencia clínica, la positividad para carcinoma en la rebiopsia (43\%) es semejante a la descrita en la literatura. Tal porcentaje de rebiopsias positivas, respalda la vigilancia clínica y el reindicar rebiopsias sistemáticas en los pacientes que presenta un PINAG aislado como la mejor actitud en la búsqueda del adenocarcinoma prostático.

\section{REFERENCIAS}

1. Stwick DG, Brawer MK. Prostatic intra-epithelial neoplasia and early invasión in prostate cancer. Cancer 1987;59(4): 788-794.

2. Chael H, Weinstein MD, Jonathan I, et al. Significance of High-Grade Prostatic Intraepithelial Neoplasia on Needle biopsy. Hum Pathol 1993;24:624-629.

3. Mc Neal JE, Bostwick DG. Intraductal dysplasia: A premalignant lesion of the prostate. Hum Pathol 1986;17(1):6471.

4. David G, Bostwick, Gian J, et al. The incidence of high grade prostatic intraepithelial neoplasia in needle biopsies. J Urol 1995; 154(5):1791-1794

5. Horninger W, Volgger H, Rogatsch H, Strohmeyer D, Steiner $\mathrm{H}$, Hobisch A et al. Predictive value of total and percent free prostate specific antigen in high grade prostatic intraepithelial neoplasia lesions: Results of the tyrol prostate specific antigen screening project. J Urol 2001;165:1143-1145.

6. Hoedemaeker RF, Kranse R, Rietbergen JB, Kruger AE, Schroder FH, van der Kwast TH. Evaluation of prostate needle biopsies in population-based screening study. The impact of borderline lesions. Cancer 1999;85(1):145-152.

7. Herranz F: Neoplasia intraepitelial prostática diagnosticada mediante biopsia transrectal. Revisión de conjunto. Actas Urol Esp 2001;25:253-263.

8. Luján M, Páez A, Romero I, et al. Significado clínico de la neoplasia intraepitelial prostática. Arch Esp Urol 2000;53: 227-229.

9. Kronz JD, Allan CH, Shaikh A, et al. Predicting Cancer Following a Diagnosis of High-Grade Prostatic Intraepithelial Neoplasia on Neddle Biopsy. Data on men With More Than One Follow -Up Biopsy. Am J Sur Pathol 2001; 25: 1079-1085.

10. Langer JE, Rovner ES, Coleman BG, Yin D, Arger PH, Malkowicz SB, et al. Strategy for repeat biopsy of patients with prostatic intraepithelial neoplasia detected by prostate needle biopsy. J Urol 1996;155:228-231.

11. Regueiro López JC, Moreno Arcas P, Leva Vallejo M, Prieto Castro R, Anglada Curado FJ, Blanco Espinosa A. et al. Incidencia de la neoplasia intraepitelial prostática de alto grado en la práctica urológica. Actas Urol Esp 2000;24: 446-451

12. Kronz JD, Shaikh AA, Epstein JI. High-Grade Prostatic Intraepithelial Neoplasia With Adjacent Small Atypical Glands on Prostate biopsy. Hum Path 2001;32:389-395.

Dra. M. Arzoz Fábregas

Passeig de la Llibertat, 15

17150 Sant Gregori (Girona)

(Trabajo recibido el 30 de diciembre 2004) 